\title{
Time-lapse coherence panels for earthen dams monitoring
}

Guilherme Zakarewicz de Aguiar¹, Susanne Tainá Ramalho Maciel1 1 Universidade de Brasília (UnB)

Copyright 2021, SBGf - Sociedade Brasileira de Geofísica.

This paper was prepared for presentation during the $17^{\text {th }}$ International Congress of the Brazilian Geophysical Society held in Rio de Janeiro, Brazil, $8-11$ November 2021 (Online Event). Contents of this paper were reviewed by the Technical Committee of the $17^{\text {th }}$ International Congress of the Brazilian Geophysical Society and do not necessarily represent any position of the SBGf, its officers or members. Electronic reproduction or storage of any part of this paper for commercial purposes without the written consent of the Brazilian Geophysical Society is prohibited.

\section{Summary}

We propose a method for continuous monitoring of earthen dams by evaluating the variation of seismic velocities using passive seismic data. The method is developed from classical velocity analysis techniques used in seismic exploration. We apply the seismic interferometry technique through the cross-correlation of passively acquired seismogram traces to calculate the coherence, given by the semblance (Neidell and Taner, 1971), for each transit time curve generated by velocity/depth pairs. The Semblance values obtained are synthesized in velocity analysis panels displayed in a time-lapse, and the blurs generated in each panel moves whenever a change in the structure happens. To test the application of the method, we used the 2D finite-difference modeler fdelmodc, by Thorbecke and Draganov (2011), which simulates long-term passive seismic acquisitions from the modeling of random sources in the subsurface. We generate simple two- and three-layer horizontal geological models and complex geological models that simulate the structure of earthen dams. It was possible to simulate processes of layer saturation and test the sensitivity of the monitoring panels to variations in seismic velocities.

\section{Results and Conclusions}

The proposed monitoring panels are sensitive to variations in seismic velocities. It was possible to notice the displacement of the blur along the time-lapse panels, which indicated the increase of $\mathrm{Vp}$ simulated in the models to reflect a saturation process of the superficial layers. The velocity and depth values that generate the highest coherence values were smaller than the parameters defined for the models. In addition, the panels indicated a decrease in the thickness of the layers, although the thickness of the layers were kept fixed in the models. These minor discrepancies might be adjusted with better parameter tunning. The method has good prospects as it is sensitive to variations in physical properties of synthetic models. Future studies will use passive data acquired in an earthen dam to test the feasibility of the method in field data. 